\title{
Isolate diffuse thickening of glomerular capillary basement membrane: a renal lesion in prediabetes?
}

\author{
Fernand Mac-Moune Lai ${ }^{1}$, Cheuk-Chun Szeto ${ }^{2}$, Paul CL Choi ${ }^{1}$, Kelvin KL Ho ${ }^{2}$, \\ Nelson LS Tang ${ }^{3}$, Kai-Ming Chow ${ }^{2}$, Philip KT Li ${ }^{2}$ and Ka-Fai To ${ }^{1}$ \\ ${ }^{1}$ Department of Anatomical \& Cellular Pathology; ${ }^{2}$ Department of Medicine $\&$ Therapeutics and \\ ${ }^{3}$ Department of Chemical Pathology, The Chinese University of Hong Kong, Prince of Wales Hospital, \\ Shatin, Hong Kong, China
}

\begin{abstract}
A total of 23 patients with proteinuria and isolated ultrastructural diffuse thickening of the glomerular capillary basement membrane were studied, focusing on the possibility of diabetes mellitus, morphometry of the capillary basement membrane, and the comparison with three other groups of patients. These included 14 patients with minimal change nephropathy (MCN), 45 patients with type II diabetes arbitrarily divided into 11 early and 34 late diabetic patients, defined, respectively, as less than 3 and over 5 years history, and 13 patients biopsied for transient mild proteinuria or hematuria, with no evidence of renal disease on follow-up were used as controls. The level of proteinuria and prevalence of hematuria were similar in patients with isolated thick basement membrane and with diabetes. Diabetic retinopathy was present in $10 \%$ of early diabetes, $69 \%$ of late diabetes, but not in isolated thick basement membrane. Kimmelstiel-Wilson nodules were seen in late diabetes, and not in other patients. Hyaline arteriosclerosis was more common in late diabetes than in early diabetes or isolated thick basement membrane. The basement membrane thickness was similar between controls $(371 \pm 17 \mathrm{~nm})$ and MCN $(345 \pm 16 \mathrm{~nm})$, between patients with isolated thick basement membrane $(482 \pm 69 \mathrm{~nm})$ and early diabetes $(457 \pm 64 \mathrm{~nm})$, but significantly thicker in isolated thick basement membrane as compared to controls and MCN. In patients with isolated thick basement membrane, the basement membrane thickness was not correlated with age, smoking, body weight, hyaline arteriosclerosis, and hypertension. However, blood tests for diabetes were positive in $20 \%$ of patients at biopsy, in $44 \%$ at 6 months and $70 \%$ at 24 months followup, while seven patients showed no evidence of diabetes on follow-up. Patients with proteinuria and isolated thick glomerular basement membrane must be differentiated from MCN for therapeutic implications, and specifically managed for its strong association with prediabetes or early diabetes.

Modern Pathology (2004) 17, 1506-1512, advance online publication, 9 July 2004; doi:10.1038/modpathol.3800219
\end{abstract}

Keywords: glomerular capillary basement membrane; prediabetes; diabetic nephropathy; diabetic glomerulosclerosis; hyperinsulinemic state

In the past few years, we have encountered an increasing number of patients undergoing renal biopsy for proteinuria whose only finding was a diffuse thickening glomerular capillary basement membrane (TGCBM) at electron microscopy. Some of these patients were managed as minimal change nephropathy (MCN), but did not respond to steroids. The significance of an isolated and diffuse TGCBM was uncertain, although the possible association

Correspondence: Dr F Mac-Moune Lai, MD, FRCPA, FHKCPath, Department of Anatomical \& Cellular Pathology, Prince of Wales Hospital, Room 34055, Shatin, Hong Kong, China.

E-mail fmlai@cuhk.edu.hk

Received 20 February 2004; revised and accepted 1 June 2004; published online 9 July 2004 with diabetes was considered; however, the fasting blood glucose or glucose tolerance test were found to be normal in the majority of cases at time of biopsy. ${ }^{1-3}$ In patients with long-standing diabetes and developing nephropathy, TGCBM is a consistent but not an isolated finding, and is associated with other renal lesions including mesangial sclerosis, Kimmelstiel-Wilson nodules, hyaline exudates, and arteriosclerosis. ${ }^{1-4}$ However, many studies from Bangstad, Osterby and Hanssen had described TGCBM in young patients with type I insulin-dependent diabetes, associated with increased mesangial matrix or mesangial volume fraction, and ascribed TGCBM as the early glomerular lesion in these young diabetic patients. ${ }^{5,6}$ Nevertheless, early renal lesions in diabetic 
patients, especially in type II diabetes remain poorly described, because of the policy to withhold biopsy, unless atypical presentation raises the suspicion of nondiabetic lesions, which may affect both the management and prognosis. ${ }^{2,3,4,7}$ Moreover, TGCBM has been described in nondiabetic patients, associated with hypertension and smoking, but the correlation of TGCBM to these factors remains to be established. ${ }^{8}$

This report examined the clinical features and follow-up of 23 patients with an isolated diffuse TGCBM. This study focused on the recognition and the approach to the diagnosis of this distinctive renal lesion. We also closely examined the possible relation such renal lesion may have with diabetes mellitus or other systemic disease on follow-up. While the long-term outcome of this lesion remains to be determined, its recognition may define the appropriate management of such patients with proteinuria. $^{9,10}$

\section{Patients and methods}

\section{Patients Selection and Clinical Parameters}

All 23 patients in this study presented with proteinuria, defined as a daily excretion of protein in excess of $0.5 \mathrm{~g} /$ day, and nine of these also had microscopic hematuria. The indications for renal biopsy included proteinuria over $1.0 \mathrm{~g} /$ day or microscopic hematuria with dysmorphic red blood cells. All 23 patients demonstrated normal light microscopy, absence of immunoreactants, and a diffuse uniform TGCBM on electron microscopy. These patients were identified between 1988 and 2001, with no history of diabetes or systemic disease, and all with at least 12 months follow-up. From the same period, we also studied 45 patients with type II diabetes and a renal biopsy for proteinuria or hematuria, divided arbitrarily into two groups: 11 patients defined as early diabetes for less than 3 years history and 34 patients defined as late diabetes with over 5 years history, all with KimmelstielWilson nodules. In total, 13 sex- and age-matched patients with a normal biopsy, performed for proteinuria under $1 \mathrm{~g} /$ day or microscopic hematuria, with no evidence of renal disease on follow-up were used as controls. We also included 14 patients with nephrotic syndrome and biopsy-confirmed MCN, who completely recovered.

We recorded the patient's sex, age, smoking history, body weight, blood pressure, retinopathy, 24-h proteinuria (measured by Modular Analytics DP, Roche Diagnostics, Switzerland), nephrotic range proteinuria (defined for a daily excretion over 3.5 g/day), hematuria, and serum creatinine. Fasting plasma glucose (FPG, measured by hexokinase method, by Modular Analytics DP, Roche Diagnostics, Switzerland) was available in all patients at the time of biopsy, except 13 patients with TGCBM in whom FPG was tested within 6 months after biopsy. When FPG was normal, an oral glucose tolerance test (OGTT), but not glycosylated hemoglobin assay, was performed to rule out diabetes. Diabetes was defined for an FPG equal to or above $126 \mathrm{mg} / \mathrm{dl}(7.0 \mathrm{mmol} / \mathrm{l})$ or an impaired glucose tolerance (IGT) with plasma glucose between $140 \mathrm{mg} / \mathrm{dl} \quad(7.8 \mathrm{mmol} / \mathrm{l})$ and $200 \mathrm{mg} / \mathrm{dl}$ (11.1 mmol/l) 2-h after a 75 -g oral glucose load. ${ }^{11}$ Impaired fasting glucose (IFG) referred to an FPG equal to or above $110 \mathrm{mg} / \mathrm{dl}(5.5 \mathrm{mmol} / \mathrm{l})$, but below $126 \mathrm{mg} / \mathrm{dl}(7.0 \mathrm{mmol} / \mathrm{l})$. Diabetic retinopathy was assessed by a physician or an ophthalmologist by fundoscopy, and defined by the presence of proliferative, preproliferative or background retinal changes, but no fluorescein examination was performed. Hypertension referred to systolic or diastolic pressures of, respectively, equal to or above 140 and $90 \mathrm{mmHg}$ and requiring antihypertensive therapy. ${ }^{12}$

\section{Pathology Assessment}

For light microscopy assessment, a minimum of 10 glomeruli and $5 \mathrm{~mm}$ renal cortex were required, and for each case pair slides were stained for hematoxylin \& eosin (H\&E), periodic acid Schiff (PAS), and periodic acid silver methenamine (PASM) with H\&E counterstain. Glomerular obsolescence (global sclerosis), mesangial hypercellularity (over four cells per mesangial area), KimmelstielWilson nodules, tubular atrophy, and hyaline arteriosclerosis were recorded. ${ }^{3}$ Immunofluorescence study included antibodies directed against human IgA, IgG, IgM, C1q, C3, and fibrin (DAKO, Glostrup, Denmark) in all cases, with kappa and lambda light chains (DAKO, Glostrup, Denmark) in TGCBM cases. All cases were processed with standard methods for electron microscopy, and examined on a CM100 Philips microscope (EDAX Inc., Eindhoven, Holland). Morphometry was carried out in a fixed setting, from image on a monitor digitalized by the Leica Quantimet $500+$ image analysis software (LEICA, Cambridge, England), at the 4600 fixed magnification. The grid intersect method was used, where 1-cm-interval grid lines image was overlapped with the capillary loop, and the intersection of grid lines with the endothelial aspect of the glomerular capillary basement membrane determined the point of measurement. ${ }^{13,14}$ The linear intercept from that point to the epithelial aspect provided a direct measure of the basement membrane in nanometer (nm) (Figure 1). This measurement was taken for all intersections between grid lines and a loop. For each case, up to 18 loops from two glomeruli were assessed to obtain a median thickness from at least 200 measurements. The morphometry was calibrated using a commercial grid with linear units, at the set magnification of 4600 and fixing a pixel unit to $8.13 \mathrm{~nm}$ in length. 


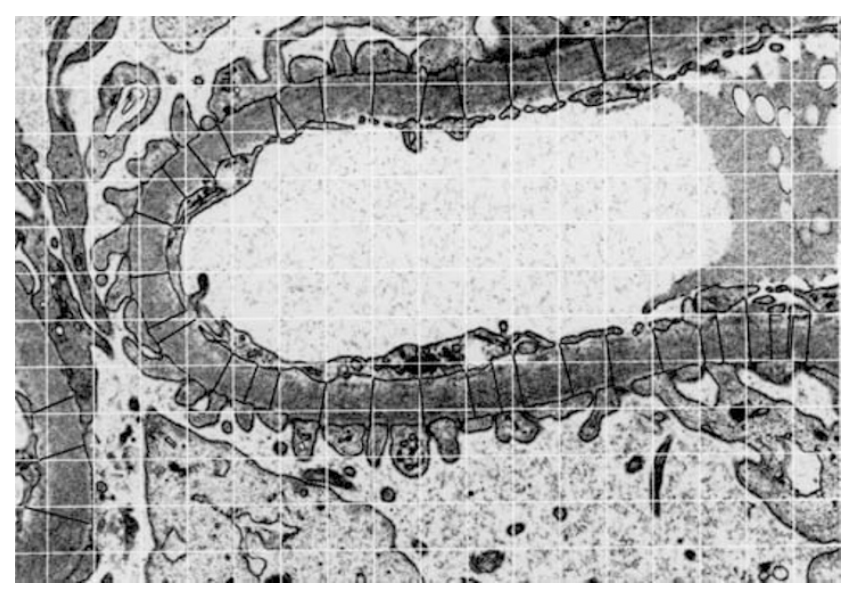

Figure 1 Grid lines (white) superposition on a glomerular capillary loop to determine the points for linear measurement of basement membrane thickness (black lines). Lead citrate and uranyl acetate, $\times 6400$.

\section{Statistical Analysis}

The statistics were analyzed using the SPSS 10.0 software for Windows (SPSS Inc., Chicago, IL, USA). The $\chi^{2}$ or Fisher's exact tests, when appropriate, were used to compare categorical variables. MannWhitney test and Spearman's correlation tests were used for nonparametric variables between groups. A $P$-value of less than 0.05 was regarded as statistically significant.

\section{Results}

Of 23 patients with TGCBM, 17 were identified in $1999,{ }^{4} 2000,{ }^{6}$ and $2001,{ }^{7}$ while only one patient in each of the 6 previous years was diagnosed, and indication of biopsy for proteinuria of $1 \mathrm{~g} /$ day or more was unchanged. The clinical features of all groups at the time of biopsy are shown in Table 1. In patients with TGCBM, the median age of 48 (22-74) years, and the male-to-female ratio of 1.09 were not significantly different from those of other groups. Two of the 23 patients were smokers (9\%), and this was similar to the prevalence of smoking of $7-15 \%$ in other groups. The median weight of the 23 patients was $57.0(42.5-77.5) \mathrm{kg}$, and was not different from that of the other groups of patients. In patients with TGCBM, proteinuria over $1 \mathrm{~g} /$ day was seen in 20 patients $(87 \%)$, nephrotic range proteinuria in six patients $(26 \%)$, hematuria in nine patients $(39 \%)$, and hypertension in $10(44 \%)$, as compared to type II diabetes patients in whom 44 $(98 \%)$ had over $1 \mathrm{~g} /$ day proteinuria, $20(44 \%)$ with nephrotic range, hematuria in 18 patients $(40 \%)$, and hypertension in 21 patients (47\%), with no significant difference observed between the two groups. Three patients with TGCBM and one with early diabetes had less than $0.5 \mathrm{~g}$ /day proteinuria. The mean proteinuria and serum creatinine were, respectively, $2.95 \mathrm{~g} /$ day and $102 \mu \mathrm{mol} / \mathrm{l}$ in TGCBM, $4.41 \mathrm{~g} /$ day and $198 \mu \mathrm{mol} / \mathrm{l}$ in diabetes, with no significant difference between the two groups. Retinopathy was not found in TGCBM patients, but in one of 10 early diabetic and 18 of 26 late diabetic patients examined. The mean proteinuria of $6.1 \mathrm{~g} /$ day in MCN was significantly higher than in TGCBM group $(P=0.01)$.

No patients with TGCBM had a history of diabetes, and only 10 of the 23 patients were tested for FPG at the time of biopsy, of which two were elevated while eight were normal (Table 2). At 6 months after biopsy, 18 patients were tested for diabetes, and eight were found to be abnormal (six new cases), including two patients each for IFG and IGT, and four with FPG over $126 \mathrm{mg} / \mathrm{dl}$. However, 10 patients demonstrated a normal FPG, including four with a normal OGTT. At 24 months follow-up, all 23 patients were tested at least twice, and 16 showed a

Table 2 Fasting plasma glucose and oral glucose tolerance test in patients with TGCBM

\begin{tabular}{lccc}
\hline & $\begin{array}{c}\text { At time of } \\
\text { biopsy }\end{array}$ & $\begin{array}{c}\text { 6 months } \\
\text { follow-up }\end{array}$ & $\begin{array}{c}\text { 24 months } \\
\text { follow-up }\end{array}$ \\
\hline Number of patients tested & 10 & 18 & 23 \\
Normal FPG $<110 \mathrm{mg} / \mathrm{dl}$ & 8 & 10 & 7 \\
IFG $110-125 \mathrm{mg} / \mathrm{dl}$ & - & 2 & 6 \\
IGT & - & 2 & 3 \\
Diabetic $\geq 126 \mathrm{mg} / \mathrm{dl}$ & 2 & 4 & 7 \\
\hline
\end{tabular}

FPG, fasting plasma glucose; TGCBM, thick glomerular basement membrane; IFG, impaired fasting glucose; IGT, impaired glucose tolerance.

Table 1 Clinical features of different groups of patients at the time of biopsy

\begin{tabular}{lcccccrr}
\hline Groups & $\begin{array}{c}\text { Patient } \\
\text { number }\end{array}$ & $\begin{array}{c}\text { Median age } \\
\text { (range) }\end{array}$ & $\begin{array}{c}\text { Proteinuria } \\
\text { (nephrotic) }\end{array}$ & $\begin{array}{c}\text { Proteinuria } \\
\text { in }(g / 24 h)\end{array}$ & Hematuria & HTN & $\begin{array}{c}\text { Diabetic } \\
\text { retinopathy }\end{array}$ \\
\hline ( $\mu$ Scrol/l)
\end{tabular}

MCN, minimal change nephropathy; TGCBM, thick glomerular capillary basement membrane; HTN, hypertension; Scr, serum creatinine. 
diabetic state $(70 \%)$, but seven patients had normal FPG, including five with OGTT. None of these seven patients had evidence of systemic disease such as hyperthyroidism, or Cushing's syndrome. All 13 controls and 14 patients with MCN had normal FPG.

On a median follow-up of 46 months, all patients with TGCBM showed persistent proteinuria, with a median of $1.5 \mathrm{~g} /$ day, and serum creatinine of $112 \mu \mathrm{mol} / \mathrm{l}$. A total of 15 patients were on angiotensin-converting enzyme inhibitor (ACEI), including all nephrotic patients and five patients treated with steroids who did not respond. Of three patients with TGCBM whose initial proteinuria was less than $0.5 \mathrm{~g} /$ day, two developed significant proteinuria between 1.5 and $2.5 \mathrm{~g} /$ day, and one remained unchanged. Patients with hypertension increased from 10 to 13, and all required therapy. A 66-year-old patient with TGCBM reached end-stage renal disease 30 months after biopsy. He presented with hypertension, hematuria, proteinuria of $2.2 \mathrm{~g} /$ day, serum creatinine of $198 \mu \mathrm{mol} / \mathrm{l}$, an FPG of $220 \mathrm{mg} / \mathrm{dl}$, and an HbA1c of $6.3 \%$. His biopsy showed hypertensive hyaline arteriosclerosis, $30 \%$ obsolescent glomeruli and $35 \%$ cortical scarring, while the viable glomeruli showed no mesangial sclerosis or Kimmelstiel-Wilson lesion, and a mean basement membrane thickness of $560 \mathrm{~nm}$. He had no retinopathy, but experienced poor control of hypertension and progressive deterioration of renal function despite therapy.

The morphology in different groups is charted in Table 3. All patients with TGCBM demonstrated normal appearing glomeruli in light microscopy, undistinguishable from MCN (Figure 2). Small number of obsolescent glomeruli with mild tubular atrophy and interstitial fibrosis were observed in a few cases of TGCBM, controls, and MCN patients. Hyaline arteriosclerosis was noted in seven (30\%) patients with TGCBM, but in no controls or MCN patients. Six (55\%) patients with early diabetes showed normal glomeruli, five (46\%) exhibited mesangial hypercellularity, and four $(26 \%)$ had hyaline arteriosclerosis associated with mild focal tubular atrophy. In patients with late diabetes, mesangial hypercellularity was observed in 22 $(65 \%)$ cases, but both Kimmelstiel-Wilson nodules and hyaline arteriosclerosis were seen in all patients (Figure 3), as well as severe tubular atrophy and interstitial fibrosis. Immunoreactants were absent in all groups of patients.

In patients with TGCBM, the thickened basement membrane was distinctive, and characterized by its uniformity, homogeneous appearance, with a faint or indistinct lamina densa (Figure 4). The degree of thickening may vary from case to case, and as the thickening process was diffuse, it may be overlooked for the unwary observer. The epithelial cell foot processes were well preserved. In contrast, all 14 patients with MCN demonstrated activated epithelial cells usually with significant fusion of the foot processes, and pronounced microvillus changes of cell membrane. The basement membrane in MCN also appeared uniform and homogeneous, but not thickened, though this observation needs morphometry to confirm. In early diabetes, the quality and thickening of the glomerular basement membrane was similar to the TGCBM group. However, in late diabetes, the thick glomerular basement membrane was not only more prominent, there were also superimposed heterogeneous lesions, including wrinkling and disruption of capillary wall, luminal collapse, nodular expansion of mesangial matrix, and electron dense amorphous exudates. The median glomerular basement membrane thickness of various groups is depicted in Table 3 , and it was $371 \pm 17 \mathrm{~nm}$ in controls, $345 \pm 16 \mathrm{~nm}$ in MCN with no statistical difference between these two groups (Table 4). The median thickness of basement membrane in TGCBM was $482 \pm 69 \mathrm{~nm}$, and showed no significant difference with that of early diabetes $457 \pm 64 \mathrm{~nm}$, but both TGCBM and early diabetes showed a significantly thicker basement membrane than controls or MCN $(P=0.000)$. In late diabetes, the median basement membrane is $640 \pm 155 \mathrm{~nm}$ thick, significantly thicker than any other groups.

In patients with TGCBM, no correlation was found between the mean thickness of the glomerular basement membrane and smoking (Mann-Whitney

Table 3 Renal lesions in different groups of patients

\begin{tabular}{|c|c|c|c|c|c|c|c|c|}
\hline $\begin{array}{l}\text { Group (number of } \\
\text { patients) }\end{array}$ & $\begin{array}{c}\text { Glomerular } \\
\text { obsolescence } \\
(\%)\end{array}$ & $\begin{array}{l}\text { Normal } \\
\text { glomeruli }\end{array}$ & $M H$ & $K W N$ & $\begin{array}{c}\text { Tubular atrophy- } \\
\text { interstitial } \\
\text { fibrosis (\%) }\end{array}$ & $H A$ & $\begin{array}{l}\text { GCBM } \\
\text { thickness } \\
\text { (nm) }\end{array}$ & $\begin{array}{l}\text { s.d. } \\
(\mathrm{nm})\end{array}$ \\
\hline Controls (13) & 2.0 & 13 & 0 & 0 & 1.0 & 0 & 371 (346-396) & 17 \\
\hline MCN (14) & 2.0 & 14 & 0 & 0 & 1.5 & 0 & $345(329-381)$ & 16 \\
\hline TGCBM (23) & 3.2 & 23 & 0 & 0 & 6.6 & 7 & $482(400-628)$ & 69 \\
\hline Diabetes (45) & 19.6 & 5 & 5 & 34 & 23.8 & 38 & $596(326-863)$ & 159 \\
\hline Early diabetes (11) & 3.7 & 6 & 5 & 0 & 7.1 & 4 & $457(373-553)$ & 64 \\
\hline Late diabetes (34) & 24.7 & 0 & 22 & 34 & 29.3 & 34 & $640(326-863)$ & 155 \\
\hline
\end{tabular}

MCN, minimal change nephropathy; TGCBM, thick glomerular capillary basement membrane; MH, mesangial hypercellularity; KWN, Kimmelstiel-Wilson nodules; HA, hyaline arteriosclerosis; GCBM, glomerular capillary basement membrane (thickness ranges in parentheses); $\mathrm{nm}$, nanometers; s.d., standard deviation. 




Figure 2 Normal appearing glomeruli in light microscopy not exhibiting the diffuse thickening of capillary basement membrane, detectable only in electron microscopy. PASM with H\&E counterstain, $\times 240$.

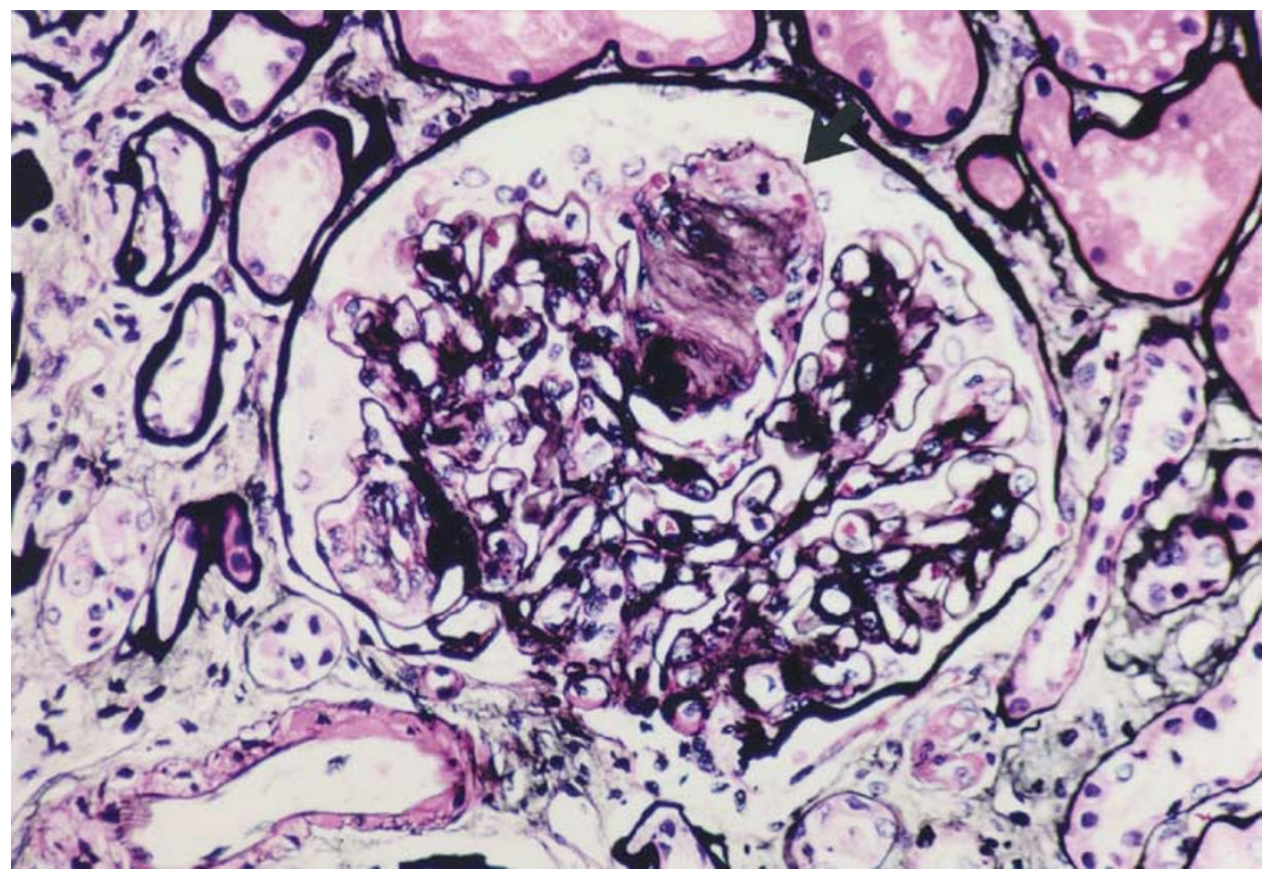

Figure 3 Diabetic glomerulosclerosis with mesangial sclerosis and Kimmelstiel-Wilson nodule (arrow). PASM with H\&E counterstain, $\times 240$.

$P=0.913$ ), body weight (Spearman's $P=0.354$ ), hypertension (Mann-Whitney $P=0.242$ ), and hyaline arteriosclerosis (Mann-Whitney $P=0.423$ ).

\section{Discussion}

TGCBM along with nodular glomerulosclerosis have been regarded as rather nonspecific lesions, as they are observed in diabetic as well as in nondiabetic patients who smoke or are hypertensive. ${ }^{8,15}$ However, TGCBM in this series was not correlated with age, body weight, smoking or hypertension, and these patients with isolated TGCBM appeared to be related to a form of chronic renal disease, substantiated by persistent proteinuria, and possibly associated with early diabetes mellitus or prediabetes. ${ }^{5,6,8}$ While patients with TGCBM and 


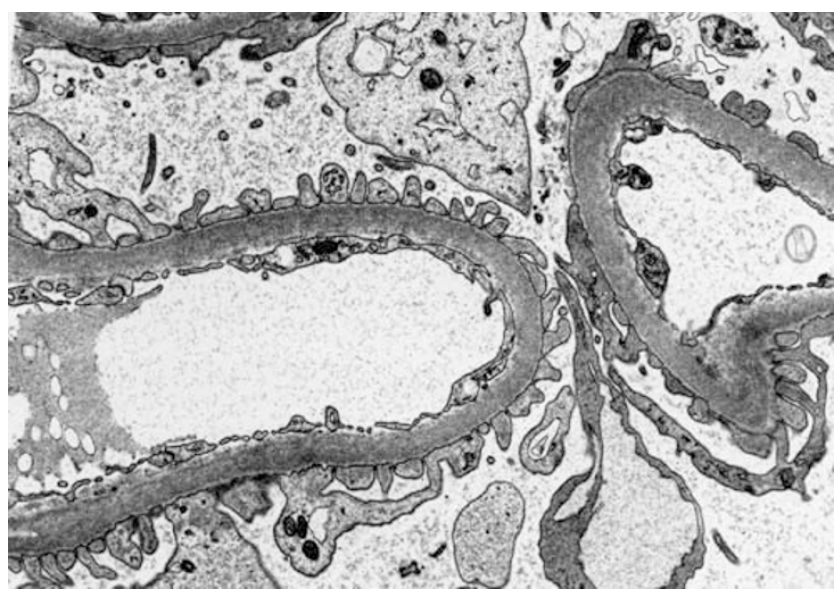

Figure 4 Thick glomerular capillary basement membrane with a uniform and homogeneous character, and inconspicuous lamina densa. Lead citrate and uranyl acetate, $\times 4800$.

Table 4 Statistical analysis of mean basement membrane thickness between groups

\begin{tabular}{lll}
\hline Controls vs MCN & 371 vs 345 & Not significant \\
Controls vs TGCBM & 371 vs 482 & $P=0.000$ \\
Controls vs early diabetics & 371 vs 457 & $P=0.000$ \\
Controls vs diabetics & 371 vs 596 & $P=0.000$ \\
TGCBM vs early diabetics & 482 vs 457 & Not significant \\
TGCBM vs late diabetics & 482 vs 640 & $P=0.000$ \\
TGCBM vs MCN & 482 vs 345 & $P=0.000$ \\
Early diabetics vs late diabetics & 457 vs 640 & $P=0.002$
\end{tabular}

Statistical $P$-values using the Mann-Whitey test; all measures of basement membrane thickness are in nanometers; MCN, minimal change nephropathy; TGCBM, thick glomerular capillary basement membrane.

proteinuria may not represent a distinct entity, this condition needs to be differentiated from other proteinuric states with normal appearing glomeruli, in particular with MCN. The lack of response to steroids experienced in the few patients may point to a nonimmune pathogenesis, and sets TGCBM apart from MCN.

The prerequisites to the diagnosis of TGCBM include both the awareness of such a possibility during electron microscopy, and the suspicion that the basement membrane is diffusely thickened; short of these the lesion would be overlooked and morphometry not carried out. An index of suspicion for TGCBM should be upheld in the investigation of patients with proteinuria, when light and immunofluorescent microscopies are unrevealing, similar to the quest to diagnose MCN or other distinctive lesions, and to consider the use of morphometry. ${ }^{13,14}$ Clearly, we do not advocate renal biopsy and tedious morphometry to establish the diagnosis of early diabetes, but this series of patients was distinctive for the actual lack of biochemical evidence of diabetes when renal manifestations appeared.
The principles of morphometry have not changed, but its practice has improved from the measurements and calculations based on microphotographs to the direct measure using softwares designed for quantitation. ${ }^{13,14}$ The direct measurement on the monitor image actually bypasses many steps involved in calculations from the photographic plates to the final prints, and thus reduces the introduction of errors. ${ }^{13,14}$ Arguably, our 'controls' may not reflect the normal population, but short of biopsy in normal individuals, we have insured that these relative controls were age- and sex-matched, had normal plasma glucose levels, and no evidence of chronic renal disease on follow-up. Based on these controls, the capillary basement membrane thickness in patients with MCN was shown to be similar, and may serve as reference to normal ranges. The capillary basement membrane thickness in patients with TGCBM was significantly increased as compared to controls, and established that TGCBM was indeed the only detectable lesion in this series of patients.

As thick capillary basement membrane is described in early diabetes, and is common in longstanding diabetes, it was only logical to monitor these patients with an isolated TGCBM for clinical and biochemical features of diabetes., ${ }^{5,6}$ Initially, however, many patients clearly did not demonstrate any supporting evidence for diabetes and a few still did not after a median follow-up of 46 months. Nonetheless, a majority of patients developed consistent abnormal blood tests in the course of followup, establishing the diagnosis of diabetes, and ascribing TGCBM to a prediabetic state. One may argue that the coexistence of TGCBM and diabetes is merely coincidental, but whether such an argument is legitimate or refutable, the recognition of an isolated TGCBM denotes a close and important link with early diabetes or prediabetes in most of these cases. . $, 6,16^{-16}$

In fact, the mechanisms involved in the development of diabetic lesions or thickening of basement membrane are poorly understood, but many processes induced by hyperglycemia have been implicated, including a deranged sorbitol pathway, excessive production of advanced glycosylation end products, and synthesis of glucoaminoglycans. ${ }^{2,10}$ As most of our patients showed no hyperglycemia at the time of biopsy, these processes unlikely contributed to the development of TGCBM. Cusumano et $a l^{16}$ recently observed glomerular enlargement and thickening of basement membrane in the rhesus monkey before the appearance of clinical overt diabetes, and correlated these renal lesions with hyperinsulinemia or the prediabetic state. Abnormal intrarenal hemodynamics and glomerular hyperfiltration have also been invoked in structural changes in incipient diabetes. ${ }^{1,10,16,17}$ In this series, we did not measure serum insulin, glomerular size, or glomerular hyperfiltration, thus their role in the development of TGCBM is not known. 
While a firm relationship between isolated TGCBM and diabetes remains to be proven, the detection of this renal lesion has several practical implications in the patients' management. This lesion, which heralds an increased risk for diabetes, may provide the opportunity to prevent its development, particularly if other risk factors like obesity or hyperlipidemia are present.,10,17 TGCBM must be differentiated from MCN, because treatment with steroids is not only ineffective in TGCBM, it may provoke diabetes. In the absence of specific therapy, our patients with TGCBM were treated with ACEI or angiotensin-receptor antagonists for proteinuria, with only partial response. Although the renoprotective effect of ACEI and angiotensin-receptor antagonists has been shown in established diabetic nephropathy, their effectiveness in prediabetes or in TGCBM patients is worth to explore. ${ }^{17-19}$ Most patients with TGCBM showed a preserved renal function on follow-up, though their long-term prognosis remains to be assessed. As for the seven patients with no evidence of diabetes or other systemic disease apart from hypertension in two, proteinuria persisted after 30 months follow-up. Irrespective of diabetes, TGCBM is a measurable lesion that can be reassessed to appraise therapy or its possible evolution.

New patients with isolated TGCBM has continued to increase, and while this may reflect our awareness for the condition, more importantly such an increase may translate the epidemic rise of type II diabetes in our region, as in other parts of the world. ${ }^{17,20}$ Such an epidemic of diabetes is bound to be paralleled by the rise of its complications, and the unheralded number of new cases from renal biopsy indicates that the health burden of such 'medical catastrophe' has also emerged in our locality. ${ }^{17,20}$ From these perspectives, even short of a firm relationship with diabetes, the recognition of TGCBM makes it worth to explore the potential application of this measurable renal lesion in the prevention and reduction of diabetic complications.

\section{References}

1 Ritz E, Stefanski A. Diabetic nephropathy in type II diabetes. Am J Kidney Dis 1996;27:167-194.

2 Mazzucco G, Bertani T, Fortunato M, et al. Different patterns of renal damage in type 2 diabetes mellitus: a multicentric study on 393 biopsies. Am J Kidney Dis 2002;39:713-720.

3 Mac-Moune Lai F, Li PKT, Pang SW, et al. Diabetic patients with IgA nephropathy and diabetic glomerulosclerosis. Mod Pathol 1993;6:684-690.

4 Olsen S, Mogensen CE. How often is NIDDM complicated with non-diabetic renal disease? An analysis of renal biopsies and the literature. Diabetologia 1996; 39:1638-1645.
5 Bangstad HJ, Osterby R, Dahl-Jorgensen K, et al. Early glomerulopathy is present in young, type 1 (insulindependent) diabetic patients with microalbuminuria. Diabetologia 1993;36:523-529.

6 Bangstad HJ, Osterby R, Rudberg S, et al. Kidney function and glomerulopathy over 8 years in young patients with Type I (insulin-dependent) diabetes mellitus and microalbuminuria. Diabetologia 2002;45: 253-261.

7 Wong TYH, Choi PCL, Szeto CC, et al. Renal outcome in type 2 diabetic patients with or without coexisting non-diabetic nephropathies. Diabetic Care 2002;25: 900-905.

8 Markowitz GS, Lin J, Valeri AM, et al. Idiopathic nodular glomerulosclerosis is a distinct clinicopathologic entity linked to hypertension and smoking. Hum Pathol 2002;33:826-835.

9 Hostetter TH. Prevention of end-stage renal disease due to type 2 diabetes. N Engl J Med 2001;345: 910-912.

10 Bertani T, Gambara V, Remuzzi G. Structural basis of diabetic nephropathy in microalbuminuric NIDDM patients: a light microscopy study. Diabetologia 1996;39:1625-1628.

11 American Diabetes Association. Clinical practice recommendations. Diabetes Care 2000;23(Suppl 1): S1-S116.

12 Joint National Comittee. The sixth report of the Joint National Committee on prevention, detection, evaluation, and treatment of high blood pressure. Arch Intern Med 1997;157:2413-2446.

13 Basta-Jovanovic G, Venkataseshan VS, Gil J, et al. Morphometric analysis of glomerular basement membrane (GBM) in thin basement membrane disease (TBMD). Clin Nephrol 1990;33:110-114.

14 Lang S, Stevenson B, Risdon RA. Thin basement membrane nephropathy as a cause of recurrent haematuria in childhood. Histopathology 1990;16: 331-337.

15 Innes A, Furness PN, Cotton RE, et al. Diabetic glomerulosclerosis without diabetes mellitus-two case reports and a review of the literature. Nephrol Dial Transplant 1992;7:642-646.

16 Cusumano AM, Bodkin NL, Hansen BC, et al. Glomerular hypertrophy is associated with hyperinsulinemia and precedes overt diabetes in aging rhesus monkeys. Am J Kidney Dis 2002;40:1075-1085.

17 Ritz E, Rychlik I, Locatelli F, et al. End-stage renal failure in type 2 diabetes: a medical catastrophe of worldwide dimensions. Am J Kidney Dis 1999;34: 795-808.

18 Lewis EJ, Hunsicker LG, Clarke WR, et al. for Collaborative Study Group. Renoprotective effect of the angiotensin-receptor antagonist irbesartan in patients with nephropathy due to type 2 diabetes. N Engl J Med 2001;345:851-860.

19 Parving HH, Lehnert $\mathrm{H}$, Brochner-Mortensen J, et al. The effect of irbesartan on the development of diabetic nephropathy in patients with type 2 diabetes. N Engl J Med 2001;345:870-878.

20 Chan JC, Ng MC, Critchley JA, et al. Diabetes mellitus a special medical challenge from a Chinese perspective. Diabetes Res Clin Pract 2001;54:S19-S27. 